\title{
Treatment of multiple subcutaneous Nocardia asteroides abscesses with ciprofloxacin and doxycycline
}

\author{
P.M.W. Bath ${ }^{1}$, K.W. Pettingale ${ }^{1}$ and J. Wade ${ }^{2}$
}

Departments of ${ }^{1}$ Medicine, and ${ }^{2}$ Medical Microbiology, King's College School of Medicine and Dentistry, Denmark Hill, London SE5 9RS, UK.

\begin{abstract}
Summary: We report on the successful use of oral ciprofloxacin and doxycycline in the treatment of multiple subcutaneous nocardial abscesses in an immunocompromised patient with active non-Hodgkin's lymphoma. This relatively inexpensive regimen allowed the patient to return home and was not associated with any significant side effects. The patient has shown no sign of relapse of her nocardial infection over an 8-month period on the above regimen.
\end{abstract}

\section{Introduction}

Co-trimoxazole is generally accepted as the drug of choice for infections caused by Nocardia asteroides. ${ }^{1}$ For co-trimoxazole resistant strains, despite their in vitro sensitivity to several other drugs, including tetracyclines, amikacin and cefuroxime, treatment regimens are less well established. We report here the successful use of the new 4-quinolone antimicrobial, ciprofloxacin, in combination with doxycycline, for the oral therapy of nocardiosis.

\section{Case report}

A 73 year old woman regularly attended the lymphoma clinic following the diagnosis in 1985 of diffuse lymphoblastic non-Hodgkin's lymphoma, which was histological grade 2 and clinical stage 4 , and which was initially treated with radiotherapy and combinationchemotherapy. Complete remission was never achieved and the histology subsequently changed to plasmacytoid lymphoma (with plasma paraprotein). In late 1987 she developed multiple painful fluctuant subcutaneous masses affecting the right axilla, right arm and left thigh. These were initially treated as extra-lymphatic lymphomatous deposits with chlorambucil and prednisolone but therapy was stopped when the axillary mass burst releasing pus which revealed Gram-positive branching rods compatible with Nocardia or Actinomyces species. Treatment was started with co-trimoxazole ( 2 capsules twice daily) and benzylpenicillin (1 megaunit 6-hourly).

The remaining abscesses were surgically drained. Ten days later culture of the pus yielded Nocardia

Correspondence: P.M.W. Bath, B.Sc., M.R.C.P. (UK)

Accepted: 28 September 1988 asteroides and benzylpenicillin was stopped. The patient was otherwise well and had no symptoms or signs of pulmonary or central nervous system disease. The chest X-ray was normal. The patient became pancytopenic and although chlorambucil-induced marrow suppression was suspected the co-trimoxazole was stopped after 7 days of treatment and substituted with intravenous cefuroxime and amikacin. After days of this regimen the in vitro sensitivities of the organism were available and were as follows: sensitive to ciprofloxacin (minimum inhibitory concentration MIC $1 \mathrm{mg} / \mathrm{l}$ ), doxycycline, amoxycillin-clavulanic acid, amikacin (MIC $2 \mathrm{mg} / \mathrm{l}$ ) and cefuroxime (MIC $1 \mathrm{mg} / \mathrm{l}$; resistant to sulphamethoxazole, trimethoprim, co-trimoxazole and amoxycillin.

Her parenteral antibiotics were then changed to oral therapy with ciprofloxacin ( $500 \mathrm{mg}$ twice daily) and doxycycline $(200 \mathrm{mg}$ then $100 \mathrm{mg}$ once daily). She became pain free and two of the three abscesses ceased draining after 10 weeks. Pus from the third and largest abscess remained sterile and it healed after 4 months of therapy. Apart from intermittent diarrhoea (Clostridium difficile toxin negative) which required codeine phosphate there were no side effects. It is planned to continue treatment for a total period of 9 months.

\section{Discussion}

Although Nocardia asteroides usually causes infection in immunocompromised patients, especially those with lymphoma, it may also infect the immunocompetent. ${ }^{2}$ Local infection may become disseminated to the lungs, central nervous system or skin when the prognosis becomes poor. Other poor prognosticators include immunosuppression from drugs or disease. ${ }^{3}$ 
The organism is usually sensitive to co-trimoxazole which is considered to be the drug of choice. ${ }^{1}$ Therapy with two agents, as in co-trimoxazole, may offer advantages and treatment is recommended for up to a year. ${ }^{4}$

Ciprofloxacin is a new 4-quinolone antibacterial agent with excellent tissue penetration and a high volume of distribution reflecting good diffusion into the extravascular space. ${ }^{5}$ It has been used successfully for the treatment of severe infections in immunocompromised patients. ${ }^{6}$ An oral preparation is available for twice daily administration and adverse effects are uncommon. In vitro studies suggest that ciprofloxacin should be effective in the treatment of nocardial infections. ${ }^{7-9}$ One case report describes the use of ciprofloxacin in the treatment of acquired immunodeficiency syndrome-related nocardial pneumonia; ${ }^{10}$ there was no improvement and therapy was changed to imipenem. Tetracycline derivatives have been used with success in the treatment of nocardia infections. The pharmacokinetics of doxycycline in particular

\section{References}

1. Bayley, S., Robinson, P.S. \& Eykin, S.J. Successful treatment of systemic nocardiosis with co-trimoxazole. $J$ Infect 1981, 3: 230-233.

2. Present, C.A., Wiernik, P.H. \& Serpick, A.A. Factors affecting survival in nocardiosis. Am Rev Resp Dis 1973, 108: 1444-1448.

3. Frazier, A.R., Rosenow, E.C. \& Roberts, G.D. Nocardiosis: a review of 25 cases occurring during 24 months. Mayo Clin Proc 1975, 50: 657-663.

4. Peabody, J.W. \& Seabury, J.H. Actinomycosis and nocardiosis. A review of basic differences in therapy. Am $J$ Med 1960, 28: 99-115.

5. Wise, R. \& Donovan, I.A. Tissue penetration and metabolism of ciprofloxacin. Am J Med 1987, 82 (suppl 4A): $103-107$.

6. Wood, M.E. \& Newland, A.C. Intravenous ciprofloxacin in the treatment of infection in immunocompromised patients. J Antimicrob Chemother 1986, 18 (suppl D): 175-178. suggest that it would be an effective agent for the treatment of soft tissue nocardia infections. ${ }^{11}$

We believe that healing of the subcutaneous abscesses and prevention of further dissemination of the organism in our patient indicate that the combination of ciprofloxacin and doxycycline may be considered for the treatment of infections due to Nocardia asteroides when co-trimoxazole cannot be used (because of resistance on the part of the organism, allergy or concern about marrow suppression). This combination constitutes a simple, oral and moderately priced regimen allowing patients to be discharged home after initial parenteral therapy has ceased.

\section{Acknowledgements}

We thank Dr A.H.C. Uttley of Dulwich Hospital for determining the antimicrobial sensitivities, Dr J. PhilpottHoward for suggesting the use of ciprofloxacin, and Professor M. Casewell for help in the preparation of the manuscript.

7. Felmingham, D., O’Hare, M.D., Robbins, M.J. et al. Comparative in vitro studies with 4-quinolone antimicrobials. Drugs Exptl Clin Res 1985, XI: 317-329.

8. Gombert, M.E., Aulicino, T.M., du Bouchet, L. \& Berkowitz, L.R. Susceptibility of Nocardia asteroides to new quinolones and beta-lactams. Antimicrob Agents Chemother 1987; 31: 2013-2014.

9. Berkey, P., Moore, D. \& Rolston, K. In vitro susceptibilities of Nocardia species to newer antimicrobial agents. Antimicrob Agents Chemother 1988, 32: $1078-1079$.

10. Shah, P.M., Just, G., Staszewski, S., Helm, E.B., Brodt, R. \& Stille, W. Ciprofloxacin und imipenem bei Nocardiose. Deutsch Med Wochenschr 1987, 112: 1101.

11. Dewsnup, D.H. \& Wright, D.N. In vitro susceptibility of Nocardia asteroides to 25 antimicrobial agents. Antimicrob Agents Chemother 1984, 25: 165-167. 